\title{
СОСТОЯНИЕ МАКРОГЕМОДИНАМИКИ И МИКРОЦИРКУЛЯЦИИ У ПАЦИЕНТОВ С ОККЛЮЗИОННО-СТЕНОТИЧЕСКИМ ПОРАЖЕНИЕМ МАГИСТРАЛЬНЫХ АРТЕРИЙ НИЖНИХ КОНЕЧНОСТЕЙ ПРИ РАЗЛИЧНЫХ ТИПАХ РЕВАСКУЛЯРИЗУЮЩИХ ВМЕШАТЕЛЬСТВ
}

\author{
( Лазаренко В.А., Бобровская Е.А., Бондарев Г.А.
}

\author{
Курский государственный медицинский университет (КГМУ) \\ Россия, 305041, Курская область, г. Курск, ул. К. Маркса, д. 3
}

\begin{abstract}
Цель исследования - изучить состояние макрогемодинамики, микроциркуляторного русла и их взаимоотношения у пациентов с окклюзионно-стенотическим поражением аорто-подвздошно-бедренного артериального сегмента до и после реваскуляризующих операций. Материалы и методы. Представлены результаты анализа состояния макрогемодинамики и микроциркуляторного русла у 330 пациентов облитерирующим атеросклерозом артерий нижних конечностей при различных типах реваскуляризующих вмешательств: бедренно-подколенное (I группа, n=140), аорто-бедренное (II группа, n=97) шунтирование, ангиопластика со стентированием подвздошных артерий (III группа, n=93). Результаты. Установлено снижение микроциркуляции в I группе - в 2,39 раза, p<0,001, во II - в 3 раза, p<0,001, в III в 2,31 раза, $\mathrm{p}<0,001$, резерва капиллярного кровотока - в I группе в 1,63 раза, $\mathrm{p}<0,001$, во II - в 2,18 раза, $\mathrm{p}<0,001$, в III - в 1,77 раза, $\mathrm{p}<0,001$ и повышение артериоло-венулярного шунтирования вне зависимости от уровня поражения, а также угнетение эндотелиального, увеличение нейрогенного механизмов регуляции микроциркуляции во всех группах. Выполнение реваскуляризующих операций не нормализует микрогемодинамику. Установлены корреляционные взаимосвязи макрогемодинамики, изменения клинического статуса и показателей микроциркуляции как базального кровотока, так и в условиях постокклюзионной гиперемии. Изменение клинического статуса после операции и показатель шунтирования имели корреляционную взаимосвязь отрицательной направленности во всех группах исследования ( $\mathrm{r}=-0,2-0,3$, $\mathrm{p}<0,05-0,01)$, а лодыжечно-плечевой индекс до и после операции и РКК - взаимосвязь положительной направленности $(\mathrm{r}=0,2-0,4, \mathrm{p}<0,05-0,001)$. Заключение. Состояние микроциркуляции оказывает влияние на изменение клинического статуса после операции, в связи с этим необходимо учитывать параметры нарушения микроциркуляции и механизмы регуляции тканевого кровотока при хирургической реваскуляризации.

Ключевые слова: облитерирующий атеросклероз артерий нижних конечностей; реваскуляризующие вмешательства; макрогемодинамика; микроциркуляторное русло; механизмы регуляции тканевого кровотока; окклюзионные функциональные пробы; лазерная допплероская флоуметрия.
\end{abstract}

Лазаренко Виктор Анатольевич - д-р. мед. наук, профессор, ректор, зав. кафедрой хирургических болезней ФПО, КГмУ, г. Курск. ORCID iD: 0000-0002-2069-7701. E-mail: azaroks@mail.ru

Бобровская Елена Анатольевна - канд. мед. наук, доцент, доцент кафедры хирургических болезней ФПО, КГМУ, г. Курск. ОRCID iD: 0000-0003-3898-5522. E-mail: ea-bobrovskaya@yandex.ru (автор, ответственный за переписку)

Бондарев Геннадий Алексеевич - д-р. мед. наук, доцент, профессор кафедры хирургических болезней ФПО, КГМУ, г. Курск. ORCID iD: 0000-0002-6761-562X. E-mail: gennadiy bondarev@mail.ru

Одной из актуальных проблем реконструктивно-восстановительных операций на аортобедренно-подколенном артериальном сегменте является рестенотический процесс в зоне анастомозов и в стенте. Частота рестенозов, по данным авторов, составляет от 6\% до 60\%, приводя к прогрессированию ишемии конечности и повторным оперативным вмешательствам [2, 3, 8, 18], при этом сам характер повторных реконструкций сложен с позиции тактики и выбора оптимального объема операции $[1,5,20]$.

Важным фактором, определяющим эффективность как открытых реконструктивных, так и эндоваскулярных вмешательств, является состояние путей притока и оттока $[1,7,9,14]$. При оценке русла оттока наиболее распространенной является балльная шкала периферического сопротивления по R. Rutherford (1997 г.) [19]. Ю.И. Казаков и соавт. разработали коэффициент состоятельности русла оттока, прогнозирующий длительность работы шунта, при двухуровневой атеросклеротической окклюзии бедренно-подколенного сегмента в стадии критической ишемии с окклюзированной по- верхностной бедренной артерией и всех магистральных артерий голени [7]. Так, при значении $0,8 \pm 0,18$ - средняя длительность проходимости шунта составляет $0,68 \pm 0,1$ года, при $1,28 \pm 0,13$ -

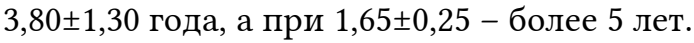

Вместе с тем оценка только состояния артерий оттока является недостаточной, поскольку емкостные свойства сосудистого русла присущи не магистральным артериям, а именно системе микроциркуляции $[1,6,15]$. Низкая пропускная способность микроциркуляторного русла, как самостоятельный фактор, в 25\% случаев приводит к развитию тромбоза при реконструктивных операциях в бедренно-подколенной зоне [15]. В работах показано, что у больных облитерирующим атеросклерозом артерий нижних конечностей отмечается нарушение функциональных возможностей микроциркуляторного русла $[11,12]$. Основной функцией микроциркуляторного русла является обеспечение тканевого гомеостаза на оптимальном уровне независимо от действия внешних и внутренних факторов [17]. 
В связи с этим целью исследования стало изучение состояния макрогемодинамики, микроциркуляторного русла и их взаимоотношения у пациентов с окклюзионно-стенотическим поражением аорто-подвздошно-бедренного артериального сегмента до и после реваскуляризующих операций.

\section{МАТЕРИАЛЫ И МЕТОДЫ ИССЛЕДОВАНИЯ}

В исследование включены 330 больных мужского пола с атеросклеротическим окклюзионностенотическим поражением аорто-подвздошнобедренного артериального сегмента, которым выполнялись реваскуляризующие операции. Вариант хирургического лечения определялся согласно общепризнанным установкам в сосудистой хирургии [13]. В зависимости от типа хирургического вмешательства больные были распределены на три группы исследования: I группу составили 140 пациентов, которым было выполнено бедренноподколенное шунтирование (БПШ), II группу 97 пациентов, которым выполнено аортобедренное шунтирование (АБШ), III группу 93 пациента, которым проведена трансбаллонная ангиопластика (ТБА) со стентированием подвздошных артерий.

Критерии включения: пациенты мужского пола с наличием облитерирующего атеросклероза аорты и артерий нижних конечностей, хронической артериальной недостаточностью нижних конечностей IIБ-III степени по классификации R. Fontaine - A.B. Покровского.

Критерии исключения: пациенты с наличием аутоиммунных заболеваний, острой и хронической патологией в стадии обострения, очагов воспаления любой локализации, заболеваниями печени, системы крови, сахарного диабета, онкологическими заболеваниями на момент обследования либо в анамнезе, декомпенсированными сердечнососудистыми заболеваниями, дегенеративными заболеваниями нервной системы, пациенты, перенесшие реконструктивные вмешательства коронарных и периферических артерий в анамнезе.

У 57,27\% (189) больных установлена ІІБ, у 42,73\% (141) III степень хронической ишемии нижних конечностей по R. Fontaine - A.B. Покровскому. Bсе пациенты в послеоперационном периоде получали традиционную консервативную терапию согласно «Национальным рекомендациям по ведению пациентов с заболеваниями артерий нижних конечностей» [13].

Помимо общеклинических методов исследования всем пациентам выполняли ультразвуковую диагностику брюшного отдела аорты и магистральных артерий нижних конечностей (LOGIQ 5 Expert, GE, Medical systems, Inc (США) с измерением лодыжечно-плечевого индекса (ЛПИ) до и после операции, аортографию и селективную артериографию нижних конечностей (ангиографический комплекс GE OEC 9800, Medical systems, Inc
(США) перед операцией с балльной оценкой состояния путей оттока.

Состояние микроциркуляции тканей исследовали методом чрескожной ЛДФ-метрии (ЛАКК 02, НПО «ЛАЗМА», Россия) в точке, расположенной на тыле стопы, в условиях физиологического покоя (базальный кровоток) и после окклюзионной пробы [10]. Выполняли амплитудночастотный анализ спектра осцилляций кровотока и оценку активных (нейрогенный, миогенный и эндотелиальный) и пассивных (дыхательный и сердечный) механизмов контроля микрогемодинамики, измерение показателя микроциркуляции (ПМ) с расчетом среднего значения в относительных перфузионных единицах (п.е.), резерва капиллярного кровотока (РКК) (\%) и показателя шунтирования (ПШ) (отн.ед.). Группу контроля для определения нормальных флоуметрических характеристик микроциркуляторного кровотока составили 20 здоровых добровольцев в возрасте

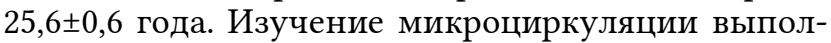
няли в группах исследования до и после реваскуляризующих вмешательств, в контрольной группе однократно.

Группы исследования были сопоставимы по полу, возрасту, продолжительности заболевания, характеру сопутствующей патологии и среднему баллу периферического сопротивления русла «оттока» (табл. 1).

Статистический анализ результатов исследования выполняли с помощью стандартного набора офисных программ Statistica 10.0, Microsoft Office, Microsoft Excel. Характер распределения анализируемых параметров оценивали с помощью критерия Колмогорова-Смирнова. Для попарного сравнения двух групп нормально распределенных данных использовали парный или непарный критерий Стьюдента, при ненормальном распределении - критерий Манн-Уитни или Вилкоксона. Количественные данные, имеющие нормальное распределение, представлены в формате $\mathrm{M} \pm \mathrm{m}$ и $\mathrm{M} \pm \sigma$, где $\mathrm{M}$ - среднее арифметическое, $\mathrm{m}$ - стандартная ошибка среднего и $\sigma$ - стандартное отклонение признака. Применялись методы параметрического корреляционного анализа, включающего определение коэффициентов корреляции с поправкой P. Фишера z. Значимость показателей р оценивали путем вычисления критериев $\mathrm{t}$ и $\mathrm{tz}$ и сравнения их значений с контрольными точками tst. Различия между группами считались статистически значимыми при уровне значимости $\mathrm{p}<0,05$.

\section{РЕЗУЛЬТАТЫ ИССЛЕДОВАНИЯ И ИХ ОБСУЖДЕНИЕ}

Выполнение оперативных вмешательств позволило добиться гемодинамического улучшения в I группе исследования в 99,3\% случаев, во II группе - в $100 \%$ случаев, в III группе - в 83,9\% случаев со значимым увеличением показателя ЛПИ после реконструктивно-восстановительных операций во всех группах исследования. 
Уровень микроциркуляторного кровотока при окклюзионно-стенотическом поражении аорты и магистральных артерий нижних конечностей был снижен у пациентов всех групп по сравнению с контрольной: в I - в 2,39 раза, $\mathrm{p}<0,001$, во II - в 3 раза, $\mathrm{p}<0,001$, в III - в 2,31 раза, $\mathrm{p}<0,001$. На фоне проведения прямой реваскуляризации конечности в I группе в объеме БПШ отмечался значимый прирост ПМ в 1,47 раза $(\mathrm{p}<0,001)$, во II группе, после АБШ - в 2,1 раза, р<0,05, в III группе, после проведения ТБА со стентированием подвздошного сегмента, в 1,35 раза, $\mathrm{p}=0,0003$ по сравнению с исходным дооперационным уровнем, что согласуется с данными литературы $[4,16]$ и указывает на реперфузию тканей. Вместе с тем значения ПМ, несмотря на макрогемодинамически эффективное восстановление кровотока в конечности, были значимо ниже, чем в контрольной группе: в I группе в 1,6 раза, $\mathrm{p}<0,001$, во II - в 1,45 раза, $\mathrm{p}<0,001$, в III - в 1,71 раза, $\mathrm{p}<0,001$. Таким образом, даже после выполнения оперативного вмешательства с восстановлением магистрального кровотока микрокровоток не достигал уровня здоровых лиц.

Установлено статистически значимое снижение РКК: в I группе в 1,63 раза, $\mathrm{p}<0,001$, во II - в 2,18 раза, $\mathrm{p}<0,001$, в III - в 1,77 раза, $\mathrm{p}<0,001$ по сравнению с контрольной группой, который после реваскуляризации увеличивался: в I группе на 15,8\%, $\mathrm{p}=0,028$, во II группе на 46,8\%, в III - на 17,3\%, p=0,04 по сравнению с дооперационным периодом, однако оставался значимо ниже по сравнению со здо-

ровыми пациентами в I группе в 1,4 раза, во II - в 1,47 раза, в III - в 1,5 раза, $\mathrm{p}<0,001$.

Амплитудно-частотный анализ базального кровотока исходно до операции показал, что у пациентов отмечались изменения активных компонентов по сравнению с контрольной группой в виде снижения эндотелиального ритма в I и III группах $(71,7 \%, \mathrm{p}<0,001$ и 71,5\%, p<0,001 соответственно) (рис. 1, 3) и практически полное отсутствие микроциркуляторного ритма в диапазоне эндотелиальной активности во II группе (рис. 2) $(-96,5 \%, p<0,001)$, а также повышения нейрогенного ритма - в I группе на 26,9\%, p=0,01, во II - на $65 \%$, $\mathrm{p}<0,001$ и в III - на $32,3 \%, p=0,039$. Изменения пассивных механизмов характеризовались снижением сердечного ритма в I $(40,6 \%, \mathrm{p}=0,002)$ и во II $(52,6 \%, \mathrm{p}<0,001)$ группах.

Выполнение реваскуляризующих операций не приводило к нормализации амплитудно-частотного спектра, несмотря на восстановление макрокровотока. Так, в I группе после БПШ при анализе базального кровотока по сравнению с дооперационным периодом наблюдался значимый прирост эндотелиальной активности $(112,1 \%, \mathrm{p}<0,001)$ и увеличение нейрогенного ритма $(12,4 \%, \mathrm{p}=0,026)$, а по сравнению с контрольной группой сохранялась та же тенденция, как и до операции: значимо низкий эндотелиальный $(39,9 \%, \mathrm{p}=0,0001)$, высокий нейрогенный $(42,7 \%, \mathrm{p}=0,011)$ и низкий сердечный $(43,9 \%, \mathrm{p}=0,0005)$ ритмы.

Таблица 1 Table 1

Клиническая характеристика пациентов

Clinical characteristics of patients

\begin{tabular}{|c|c|c|c|c|}
\hline $\begin{array}{c}\text { Показатели } \\
\text { Parameter }\end{array}$ & $\begin{array}{l}\text { I группа } \\
\text { I group }\end{array}$ & $\begin{array}{l}\text { II группа } \\
\text { II group }\end{array}$ & $\begin{array}{l}\text { III группа } \\
\text { III group }\end{array}$ & $\begin{array}{c}\text { Р между группами } \\
\text { P between groups }\end{array}$ \\
\hline $\begin{array}{l}\text { Возраст, лет } \\
\text { Age, years }\end{array}$ & $58.64 \pm 7.73$ & $56.82 \pm 6.69$ & $57.27 \pm 7.23$ & $\begin{array}{l}\text { PI-II }=0.061 \\
\text { PI-III }=0.174 \\
\text { PII-III }=0.661\end{array}$ \\
\hline $\begin{array}{l}\text { Средняя длительность заболевания, мес. } \\
\text { The average duration of the disease, months }\end{array}$ & $43.53 \pm 3.42$ & $52.65 \pm 3.07$ & $47.64 \pm 4.12$ & $\begin{array}{l}\text { PI-II }=0.06 \\
\text { PI-III }=0.44 \\
\text { PII-III }=0.33\end{array}$ \\
\hline $\begin{array}{l}\text { ИБС, абс. (\%) } \\
\text { Ischemic heart disease, abs. (\%) }\end{array}$ & $82(58.6 \%)$ & $53(54.6 \%)$ & $48(51.6 \%)$ & $\begin{array}{l}\mathrm{PI}-\mathrm{II}=0.55 \\
\mathrm{PI}-\mathrm{III}=0.29 \\
\mathrm{PII}-\mathrm{III}=0.67\end{array}$ \\
\hline $\begin{array}{l}\text { Гипертоническая болезнь, абс. (\%) } \\
\text { Hypertension, abs. (\%) }\end{array}$ & $98(70 \%)$ & $71(73.2 \%)$ & $75(80.6 \%)$ & $\begin{array}{l}\mathrm{PI}-\mathrm{II}=0.59 \\
\mathrm{PI}-\mathrm{III}=0.07 \\
\mathrm{PII}-\mathrm{III}=0.22\end{array}$ \\
\hline $\begin{array}{l}\text { Хроническая обструктивная болезнь легких, } \\
\text { абс. (\%) } \\
\text { Chronic obstructive pulmonary disease, abs. (\%) }\end{array}$ & $24(17.1 \%)$ & $21(21.6 \%)$ & $14(15 \%)$ & $\begin{array}{l}\text { PI-II }=0.39 \\
\text { PI-III }=0.67 \\
\text { PII-III }=0.24\end{array}$ \\
\hline $\begin{array}{l}\text { ОНМК в анамнезе, абс. (\%) } \\
\text { Acute disturbance of cerebral circulation in the anamnesis, } \\
\text { abs. (\%) }\end{array}$ & $1(0.7 \%)$ & $1(1.03 \%)$ & $4(4.3 \%)$ & $\begin{array}{l}\text { PI-II }=0.79 \\
\text { PI-III }=0.06 \\
\text { PII-III }=0.16\end{array}$ \\
\hline $\begin{array}{l}\text { Средний балл состояния путей оттока } \\
\text { The average score of the peripheral resistance of the "out- } \\
\text { flow" channel }\end{array}$ & $3.99 \pm 0.16$ & $4.44 \pm 0.20$ & $4.05 \pm 0.36$ & $\begin{array}{l}\text { PI-II }=0.071 \\
\text { PI-III }=0.846 \\
\text { PII-III }=0.343\end{array}$ \\
\hline
\end{tabular}




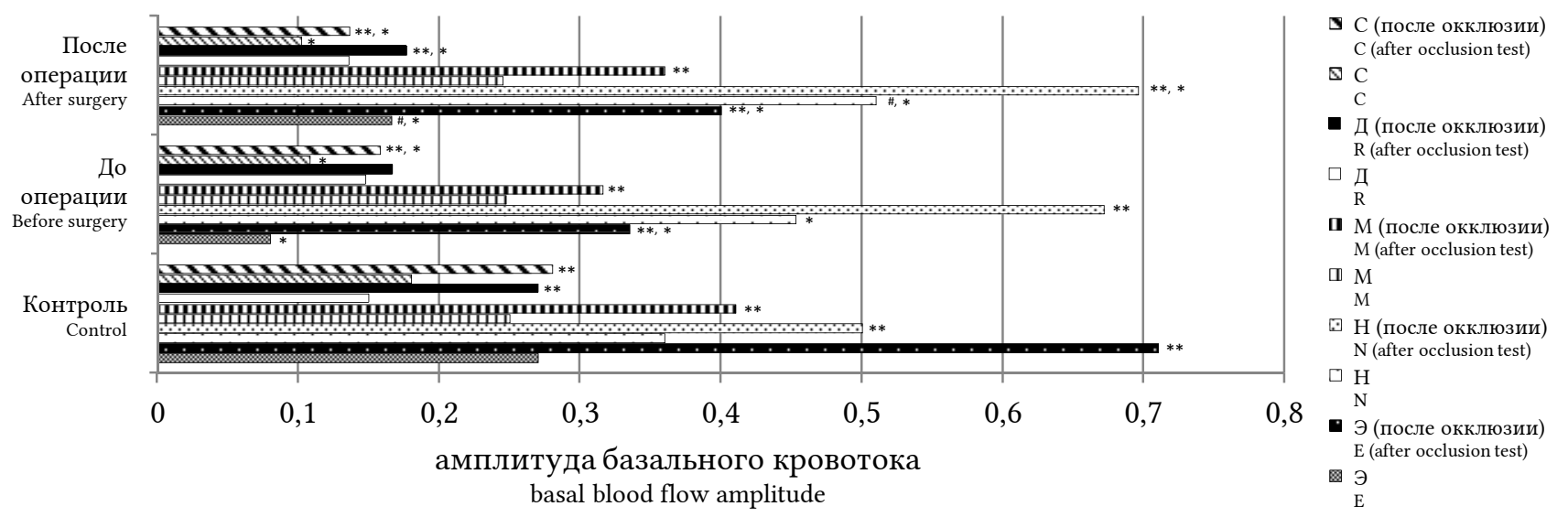

Рис. 1. Функциональная активность регуляторных механизмов модуляции кровотока у пациентов І группы исследования.

Примечания (здесь и на рис. 2, 3): регуляторные механизмы микроциркуляции: Э - эндотелиальный, H - нейрогенный, $\mathrm{M}$ - миогенный, Д - дыхательный, C - сердечный; * - $<0,05$ по отношению к контрольной группе, ${ }^{\#}-\mathrm{p}<0,05$ по отношению к группе пациентов до операции, ${ }^{* *}-\mathrm{p}<0,05$ значимость различий значений после проведения окклюзионной пробы по отношению к значению базального кровотока.

Fig. 1. Functional activity of regulatory mechanisms of blood flow modulation in group I patients

Note (here and in Fig. 2, 3): regulatory mechanisms of microcirculation: $\mathrm{E}$ - endothelial, $\mathrm{N}$ - neurogenic, $\mathrm{M}$ - myogenic, $\mathrm{R}-$ respiratory, $\mathrm{C}-$ cardiac; ${ }^{*}-\mathrm{p}<0.05$ in relation to the control group, ${ }^{*}-\mathrm{p}<0.05$ in relation to the group of patients before surgery, ${ }^{* *}-\mathrm{p}<0.05$ significance of differences in values after occlusion test in relation to the value of basal blood flow.
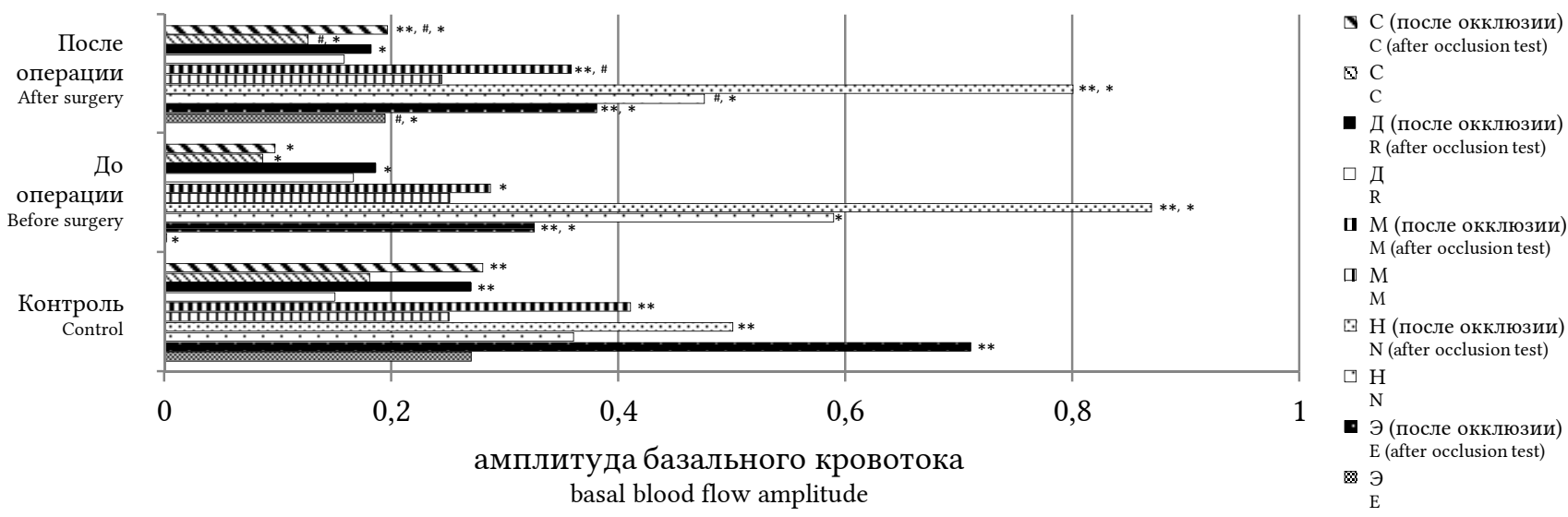

Рис. 2. Функциональная активность регуляторных механизмов модуляции кровотока у пациентов II группы исследования.

Fig. 2. Functional activity of regulatory mechanisms of blood flow modulation in group II patients.

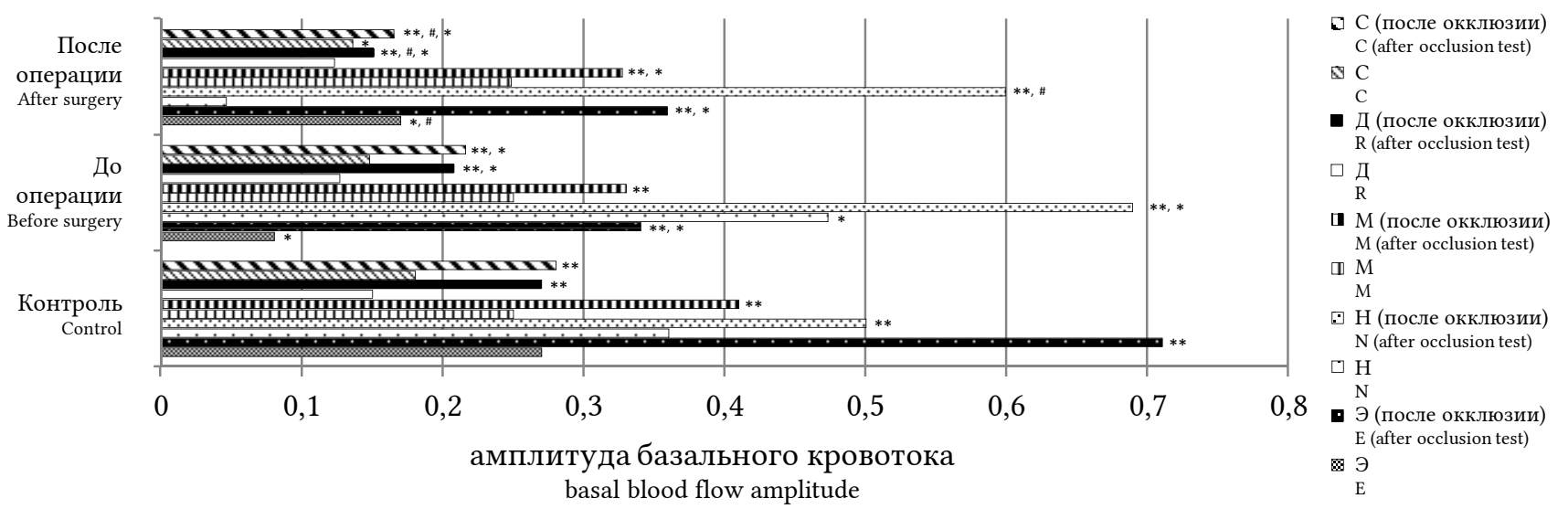

Рис. 3. Функциональная активность регуляторных механизмов модуляции кровотока у пациентов III группы исследования.

Fig. 3. Functional activity of regulatory mechanisms of blood flow modulation in group III patients. 
Bo II группе после АБШ при амплитудночастотном анализе ЛДФ-грамм базального кровотока по сравнению с показателями до оперативного вмешательства установлено значимое увеличение эндотелиального (в 21 раз, $\mathrm{p}<0,001$ ), снижение нейрогенного $(19,5 \%, \mathrm{p}=0,0002)$ и повышение сердечного $(46,9 \%, \mathrm{p}<0,001)$ ритмов, вместе с тем значимых изменений миогенного ритма после операции не наблюдалось, а по сравнению с контрольной группой, регистрируемый эндотелиальный ритм в послеоперационном периоде был значимо ниже контрольной группы $(29,9 \%, \mathrm{p}=0,045)$, сохранялись высокие значения нейрогенного $(19,5 \%, \mathrm{p}=0,017)$ и низкие сердечного $(30,4 \%, \mathrm{p}=0,008)$ ритмов.

У пациентов III группы по сравнению с исходным дооперационным уровнем значимых изменений в динамике микроциркуляторных ритмов после ТБА со стентированием подвздошного сегмента не наблюдалось, за исключением выраженного прироста эндотелиальной активности в 2,18 раза, $\mathrm{p}<0,001$ и по сравнению с контрольной группой сохранялись низкие значения эндотелиального $(38,5 \%, \mathrm{p}=0,0002)$ и сердечного $(25,2 \%$, $\mathrm{p}=0,05)$ ритмов, а нейрогенный компонент был повышен $(28,2 \%, \mathrm{p}=0,056)$, однако не был статистически значимым.

Проведенные исследования показали, что амплитуда колебаний в миогенном частотном диапазоне у больных не претерпевает значимых изменений в послеоперационном периоде, в отличие от работ Н.Н. Стрельцовой и соавт, наблюдавших увеличение роли как эндотелиальных, так и миогенных механизмов контроля после эндоваскулярной реваскуляризации конечности, наряду со снижением амплитуды в нейрогенном частотном диапазоне на 59,4\% [16].

Изменения в постокклюзионной вейвлетграмме, с одной стороны, характеризовались зна- чимым повышением амплитуды колебаний активных механизмов модуляции микрокровотока, как исходно в до операционном периоде (эндотелиального, нейрогенного, миогенного в I и III группах, эндотелиального и нейрогенного во II группе), так и после операции (во всех группах), указывающих на сохранение функциональности, с другой стороны, изменение пассивных механизмов модуляции микрокровотока в виде увеличения амплитуды сердечных (в I и III группах) и дыхательных колебаний (в III группе) исходно до операции, так и после операции (сердечных ритмов во всех группах и дыхательных ритмов в I и III группах), что может свидетельствовать о нарушениях венозного оттока и усилении венозного застоя при увеличении кровотока.

ПШ, характеризующий соотношение шунтового и нутритивного кровотока, демонстрировал исходно высокий уровень до операции (рис. 4), превышая контрольные значения в I группе на $31,9 \%, \mathrm{p}<0,001)$, во II - на $48,4 \%, \mathrm{p}<0,001)$, в III - на $28,1 \%, \mathrm{p}=0,001$ с приростом ПШ в постокклюзионной вейвлет-грамме $(14,6 \%, \mathrm{p}<0,001,16,2 \%, \mathrm{p}=0,008$, $17,2 \%, \mathrm{p}<0,001$ соответственно). После прямой реваскуляризации ПШ снижался относительно дооперационных значений в I группе $(20,1 \%, \mathrm{p}<0,001)$, не отличаясь от контрольной группы $(\mathrm{p}=0,41)$, и во II группе $(14,7 \%$, p=0,0002), оставаясь высоким и превышая показатель контрольной группы на $26,7 \%, \mathrm{p}=0,001)$. В III группе после операции имелась некоторая недостоверная тенденция к снижению ПШ (7,6\%, p=0,06), превышая контрольные значения на $18,3 \%, \mathrm{p}=0,02$. Наблюдался прирост показателя после проведения окклюзионной пробы во всех хирургических группах $(23,2 \%, \mathrm{p}<0,001$, $11,8 \%, \mathrm{p}=0,003,14,6 \%, \mathrm{p}=0,001$ в I, II и III группах соответственно), что не являлось нормальной реакцией микрососудистого русла и свидетельствовало о повышении артериоло-венулярного сброса.

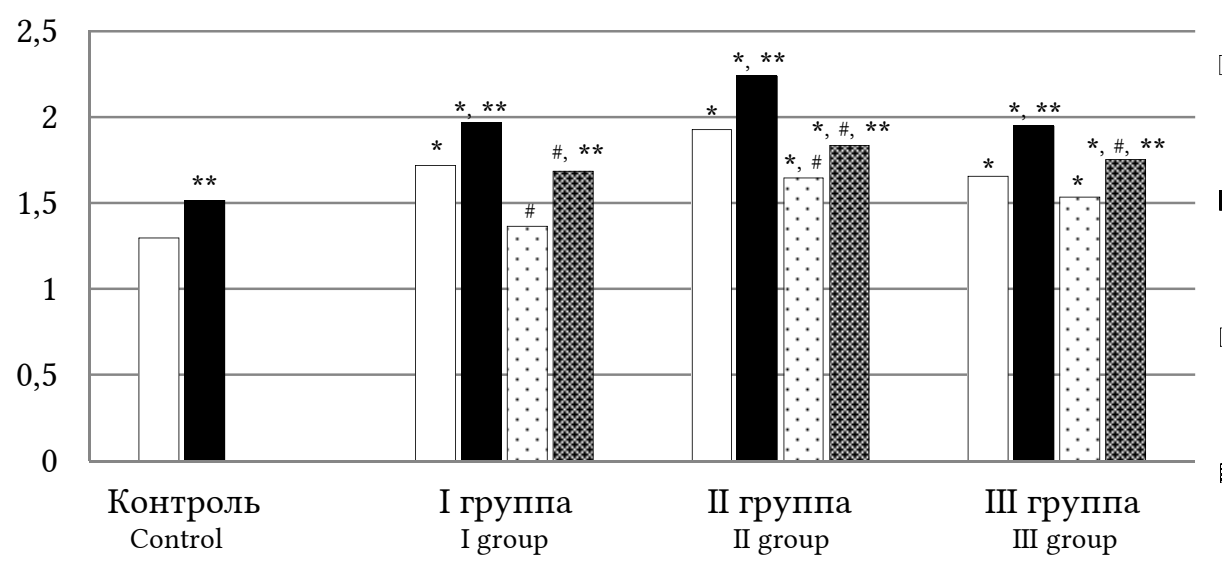

Базальный кровоток Basal blood flow

После окклюзионной пробы After the occlusion test

Базальный кровоток после оперции Basal blood flow after surgery

После операции и после окклюзионной пробы After surgery and after the occlusion test

Рис. 4. Динамика ПШ (отн. ед.) в группах исследования.

Примечание: * - p<0,05 по отношению к контрольной группе, ${ }^{\#}-\mathrm{p}<0,05$ по отношению к группе пациентов до операции, ${ }^{* *}-\mathrm{p}<0,05$ по отношению к значению базального кровотока.

Fig 4. Dynamics of bypass index (relative units) in study groups.

Note: ${ }^{*}-\mathrm{p}<0.05$ in relation to the control group, ${ }^{*}-\mathrm{p}<0.05$ in relation to the group of patients before surgery, ${ }^{* *}-\mathrm{p}<0.05$ in relation to basal blood flow. 
Таблица 2

Table 2

Корреляционные взаимосвязи макрогемодинамики,

изменения клинического статуса и данных микроциркуляции

Correlations of macrohemodynamics, changes in clinical status and microcirculation data

\begin{tabular}{|c|c|c|c|c|c|c|c|c|c|c|}
\hline \multirow{2}{*}{$\begin{array}{c}\text { Показатели } \\
\text { Parameter }\end{array}$} & \multirow{2}{*}{$\begin{array}{c}\text { Группы } \\
\text { Groups }\end{array}$} & \multirow{2}{*}{$\begin{array}{l}\text { До/ } \\
\text { После } \\
\text { before/ } \\
\text { after }\end{array}$} & \multirow{2}{*}{$\begin{array}{l}\text { ПM } \\
\text { The mi- } \\
\text { crocircula- } \\
\text { tion index }\end{array}$} & \multicolumn{5}{|c|}{$\begin{array}{c}\text { Регуляторные механизмы } \\
\text { микроциркуляции } \\
\text { Regulatory mechanisms of microcirculation }\end{array}$} & \multirow{2}{*}{$\begin{array}{l}\text { ПШ } \\
\text { Bypass } \\
\text { index }\end{array}$} & \multirow{2}{*}{$\begin{array}{l}\text { PKK } \\
\text { Capillary } \\
\text { blood flow } \\
\text { reserve }\end{array}$} \\
\hline & & & & $\begin{array}{l}\ni \\
\mathrm{E}\end{array}$ & $\begin{array}{l}\mathrm{H} \\
\mathrm{N} \\
\end{array}$ & $\begin{array}{l}\mathrm{M} \\
\mathrm{M} \\
\end{array}$ & $\begin{array}{l}\text { Д } \\
\mathrm{R} \\
\end{array}$ & $\begin{array}{l}\mathrm{C} \\
\mathrm{C} \\
\end{array}$ & & \\
\hline \multirow{6}{*}{$\begin{array}{l}\text { Состояние } \\
\text { путей } \\
\text { оттока } \\
\text { The index of } \\
\text { peripheral } \\
\text { resistance of the } \\
\text { outflow }\end{array}$} & \multirow{2}{*}{ I } & $\begin{array}{c}\text { मо } \\
\text { before }\end{array}$ & $-0.19^{*}$ & 0.03 & 0.03 & $-0.23^{* *}$ & $0.20^{*}$ & 0.11 & 0.12 & \multirow{2}{*}{-0.15} \\
\hline & & $\begin{array}{l}\Pi / \mathrm{o} \\
\text { after } \\
\end{array}$ & & 0.00 & 0.06 & -0.04 & -0.03 & -0.06 & 0.09 & \\
\hline & \multirow{2}{*}{ II } & $\begin{array}{c}\text { मо } \\
\text { before }\end{array}$ & $-0.31^{* *}$ & -0.06 & 0.14 & $-0.24^{*}$ & 0.03 & -0.15 & $0.25^{*}$ & -0.19 \\
\hline & & $\begin{array}{l}\Pi / 0 \\
\text { after }\end{array}$ & & -0.01 & 0.07 & $-0.22^{*}$ & 0.00 & -0.19 & 0.05 & \\
\hline & \multirow{2}{*}{ III } & $\begin{array}{c}\text { मо } \\
\text { before }\end{array}$ & 0.10 & -0.01 & 0.06 & -0.09 & 0.02 & 0.07 & 0.04 & \\
\hline & & $\begin{array}{l}\Pi / \mathrm{O} \\
\text { after }\end{array}$ & & 0.06 & 0.12 & 0.06 & 0.00 & 0.04 & 0.01 & 0.13 \\
\hline \multirow{6}{*}{$\begin{array}{c}\text { ЛПИ } \\
\text { до } \\
\text { операции } \\
\text { ABI before sur- } \\
\text { gery }\end{array}$} & \multirow{2}{*}{ I } & $\begin{array}{c}\text { до } \\
\text { before }\end{array}$ & $0.29^{* * *}$ & 0.08 & $-0.22^{* *}$ & $0.21^{*}$ & $-0.18^{*}$ & -0.11 & $-0.24^{* *}$ & \\
\hline & & $\begin{array}{l}\Pi / 0 \\
\text { after } \\
\end{array}$ & & -0.00 & -0.14 & -0.02 & -0.07 & -0.03 & $-0.20^{*}$ & $0.25^{* *}$ \\
\hline & \multirow{2}{*}{ II } & $\begin{array}{c}\text { до } \\
\text { before }\end{array}$ & $0.46^{* * *}$ & 0.13 & $-0.29^{* *}$ & $0.30^{* *}$ & 0.18 & 0.16 & $-0.29^{* *}$ & \\
\hline & & $\begin{array}{l}\Pi / \mathrm{O} \\
\text { after }\end{array}$ & & 0.04 & -0.11 & 0.17 & 0.04 & -0.05 & -0.06 & $0.43^{* * *}$ \\
\hline & \multirow{2}{*}{ III } & $\begin{array}{c}\text { до } \\
\text { before }\end{array}$ & $0.29^{* *}$ & $0.46^{* * *}$ & $-0.24^{*}$ & 0.03 & 0.04 & -0.07 & $-0.41^{* * *}$ & \\
\hline & & $\begin{array}{l}\Pi / \mathrm{O} \\
\text { after }\end{array}$ & & $0.51^{* * *}$ & 0.09 & 0.14 & 0.05 & -0.16 & $-0.25^{*}$ & $0.25^{*}$ \\
\hline \multirow{6}{*}{$\begin{array}{c}\text { ЛПИ } \\
\text { после } \\
\text { операции } \\
\text { ABI after } \\
\text { surgery }\end{array}$} & \multirow{2}{*}{ I } & $\begin{array}{c}\text { मо } \\
\text { before }\end{array}$ & $0.31^{* * *}$ & $0.19^{*}$ & -0.13 & $0.19^{*}$ & -0.13 & -0.10 & $-0.22^{* *}$ & \\
\hline & & $\begin{array}{l}\Pi / \mathrm{O} \\
\text { after }\end{array}$ & & 0.01 & $-0.19^{*}$ & 0.06 & 0.06 & $-0.19^{*}$ & $-0.23^{* *}$ & $0.19^{*}$ \\
\hline & \multirow{2}{*}{ II } & $\begin{array}{c}\text { до } \\
\text { before }\end{array}$ & -0.01 & 0.19 & -0.08 & 0.04 & -0.16 & -0.06 & -0.19 & \\
\hline & & $\begin{array}{l}\Pi / 0 \\
\text { after } \\
\end{array}$ & & 0.03 & $-0.20^{*}$ & 0.15 & $-0.26^{* *}$ & 0.05 & $-0.23^{*}$ & $0.29^{* *}$ \\
\hline & \multirow{2}{*}{ III } & $\begin{array}{c}\text { до } \\
\text { before }\end{array}$ & $0.45^{* * *}$ & $0.34^{* *}$ & $-0.26^{*}$ & $0.31^{* *}$ & 0.01 & $-0.21^{*}$ & $-0.41^{* * *}$ & \\
\hline & & $\begin{array}{l}\Pi / \mathrm{o} \\
\text { after }\end{array}$ & & $0.51^{* * *}$ & -0.11 & $0.46^{* * *}$ & -0.06 & $-0.30^{* *}$ & $-0.39^{* * *}$ & $0.34^{* *}$ \\
\hline \multirow{6}{*}{$\begin{array}{c}\text { Изменение } \\
\text { клиниче- } \\
\text { ского } \\
\text { статуса } \\
\text { после } \\
\text { операции } \\
\text { Change } \\
\text { in clinical } \\
\text { status after } \\
\text { surgery }\end{array}$} & \multirow{2}{*}{ I } & $\begin{array}{c}\text { до } \\
\text { before }\end{array}$ & $0.30^{* * *}$ & $0.18^{*}$ & -0.10 & 0.13 & -0.16 & -0.16 & $-0.19^{*}$ & \\
\hline & & $\begin{array}{l}\Pi / 0 \\
\text { after }\end{array}$ & & 0.08 & -0.14 & 0.09 & -0.01 & $-0.17^{*}$ & $-0.19^{*}$ & 0.08 \\
\hline & \multirow{2}{*}{ II } & $\begin{array}{c}\text { मо } \\
\text { before }\end{array}$ & -0.03 & 0.13 & -0.15 & 0.01 & -0.10 & -0.06 & $-0.21^{*}$ & \\
\hline & & $\begin{array}{l}\Pi / \mathrm{O} \\
\text { after }\end{array}$ & & 0.07 & -0.19 & 0.13 & $-0.23^{*}$ & 0.05 & $-0.21^{*}$ & $0.28^{* *}$ \\
\hline & \multirow{2}{*}{ III } & $\begin{array}{c}\text { до } \\
\text { before }\end{array}$ & $0.34^{* *}$ & $0.33^{* *}$ & $-0.29^{* *}$ & $0.22^{*}$ & -0.03 & -0.18 & $-0.33^{* *}$ & \\
\hline & & $\begin{array}{l}\Pi / 0 \\
\text { after }\end{array}$ & & $0.34^{* *}$ & -0.18 & $0.33^{* *}$ & -0.07 & $-0.33^{* *}$ & $-0.29^{* *}$ & $0.29^{* *}$ \\
\hline
\end{tabular}

Примечание: * - $<<0,05,{ }^{* *}-\mathrm{p}<0,01,{ }^{* * *}-\mathrm{p}<0,001$; до - базальный кровоток (до окклюзии), п/о - после окклюзионной пробы; Э - эндотелиальный, Н - нейрогенный, $\mathrm{M}$ - миогенный, Д - дыхательный, C - сердечный механизмы микроциркуляции.

Note: ${ }^{*}-\mathrm{p}<0.05,{ }^{* *}-\mathrm{p}<0.01,{ }^{* * *}-\mathrm{p}<0.001$; before - basal blood flow (before the occlusion test), after - after the occlusion test; $\mathrm{E}-$ endothelial, $\mathrm{N}$ - neurogenic, $\mathrm{M}$ - myogenic, $\mathrm{R}$ - respiratory, C - cardiac mechanisms of microcirculation; ABI - ankle-brachial pressure index. 
Корреляционные взаимосвязи макрогемодинамики, изменения клинического статуса и данных микроциркуляции представлены в таблице 2 . Как видно из таблицы 2, между показателями, характеризующими состояние макрогемодинамики и показателями микроциркуляции как базального кровотока, так и в условиях постокклюзионной гиперемии установлены значимые корреляционные взаимосвязи в группах исследования, что свидетельствует о том, что макро- и микроциркуляция имеют сопряженное значение в регуляции гемодинамики.

Результаты корреляционного анализа позволили установить взаимосвязи состояния путей оттока и показателя микроциркуляции базального кровотока в I и II группах ( $\mathrm{r}=-0,19, \mathrm{p}<0,05$ и $\mathrm{r}=-0,31$, $\mathrm{p}<0,01)$, миогенного компонента в I $(\mathrm{r}=-0,23, \mathrm{p}<0,01)$ и II группе (как до $(\mathrm{r}=-0,24, \mathrm{p}<0,05)$, так и после $(\mathrm{r}=-$ $0,22, \mathrm{p}<0,05)$ окклюзии), а также дыхательного компонента микроциркуляции в I группе $(\mathrm{r}=0,20$, $\mathrm{p}<0,05)$ и ПШ во II группе $(\mathrm{r}=0,25, \mathrm{p}<0,05)$. Обращает внимание, что в III группе не установлено значимых корреляционных связей состояния путей оттока и параметров микроциркуляции. Увеличение периферического сопротивления русла оттока сопровождается увеличением ПШ и, как следствие, ухудшением нутритивного кровотока при поражении аорто-подвздошного и бедренноподколенного артериального сегмента.

Следует отметить, что изменения клинического статуса после операции и ПШ как базального кровотока, так и после окклюзионной пробы имели тесную корреляционную взаимосвязь отрицательной направленности во всех группах исследования $(\mathrm{r}=-0,2-0,3, \mathrm{p}<0,05-0,01)$, а ЛПИ до и после операции и РКК - корреляционную взаимосвязь прямой направленности ( $\mathrm{r}=0,2-0,4, \mathrm{p}<0,05-0,001)$.

Таким образом, изучение состояния макрогемодинамики и микроциркуляции у пациентов с облитерирующим атеросклерозом аорты и магистральных артерий нижних конечностей до и после различных типов реваскуляризующих вмешательств позволило сделать следующие выводы:

1. У пациентов с окклюзионно-стенотическим поражением магистральных артерий нижних конечностей установлено снижение показателя микроциркуляции, РКК и повышение артериоловенулярного шунтирования вне зависимости от уровня поражения, с угнетением эндотелиального, увеличением нейрогенного механизмов регуляции микроциркуляции во всех группах исследования и снижением пульсового кровенаполнения в I и II группах по сравнению с контрольной группой.

2. Выполнение реваскуляризующих операций не нормализует микрогемодинамику, проявляющуюся увеличением нейрогенного компонента (в I группе) на фоне отсутствия динамики миогенной активности во всех группах по сравнению с данными до операции и сохраняющимся угнетением эндотелиальной активности (во всех группах), повышением нейрогенной активности (в I и II груп- пах) и снижением сердечного ритма (во всех группах) по сравнению с контрольной группой.

3. Установлено, что состояние макро и микроциркуляции имеет сопряженное значение в регуляции гемодинамики. Состояние микроциркуляции оказывает влияние на изменение клинического статуса после операции, в связи с этим необходимо учитывать параметры нарушения микроциркуляции и механизмы регуляции тканевого кровотока при хирургической реваскуляризации.

\section{КОНФЛИКТ ИНТЕРЕСОВ}

Авторы декларируют отсутствие явных и потенциальных конфликтов интересов, связанных с публикацией настоящей статьи.

\section{ИСТОЧНИКИ ФИНАНСИРОВАНИЯ}

Авторы заявляют об отсутствии финансирования.

\section{СООТВЕТСТВИЕ ПРИНЦИПАМ ЭТИКИ}

Исследование одобрено Региональным этическим комитетом при Курском государственном медицинском университете (протокол № 1 от 21.01.2012 г.). Каждым пациентом было подписано индивидуальное согласие на участие в исследовании.

\section{ЛИТЕРАTУPA/REFERENCES}

1. Белов Ю.В., Виноградов О.А., Ульянов Н.Д., Дзюндзя А.Н. Прогнозирование результатов реваскуляризирующих операций на артериях нижних конечностей на основе методов оценки регионарного кровотока. Кардиология и сердечно-сосудистая хирургия. 2014; 7(5): 62-67. [Belov Iu.V., Vinogradov O.A., Ul'ianov N.D., Dziundzia A.N. Prognosis of revascularization surgery results on lower limbs arteries on the basis of assessment of regional blood flow. Kardiologiya $i$ serdechno-sosudistaya khirurgiya. 2014.7(5): 62-67 (in Russ.)]

2. Березовская Г.А., Ганюков В.И., Карпенко М.А. Рестеноз и тромбоз внутри стента: патогенетические механизмы развития и прогностические маркеры. Российский кардиологический журнал. 2012; 17(6): 91-95. [Berezovskaya G.A., Ganyukov V.I., Karpenko M.A. Restenosis and in-stent stenosis: pathogenetic mechanisms and prognostic markers. Rossiyskiy kardiologicheskiy zhurnal. 2012; 17(6): 91-95 (in Russ.)]

3. Бурков Н.Н., Казанцев А.Н., Тарасов Р.С., Ануфриев А.И., Барбараш Л.С. Предикторы неблагоприятных исходов реконструктивных вмешательств на аортобедренном сегменте в отдаленном периоде наблюдения. Грудная и сердечно-сосудистая хирургия. 2018; 60(1): 55-60. [Burkov N.N., Kazantsev A.N., Tarasov R.S., Anufriyev A.I., Barbarash L.S. Predictors of adverse reasons of reconstructive interventions on the aorto-femoral segment in a remote period of observation. Grudnaya i serdechno-sosudistaya khirurgiya. 2018; 60(1): 55-60 (in Russ.)]

4. Васильев А.П., Стрельцова Н.Н., Саламова Л.А. Функциональное состояние микрогемоциркуляции кожи у больных облитерирующим атеросклерозом артерий нижних конечностей по данным лазерной допплеровской флоуметрии. Регионарное кровообращение и микроциркуляция. 2017; 16(4): 35-41 [Vasiliev A.P., Streltsova N.N., Salamova L.A. Functionality of skin microhemocirculation in patients with obliterating 
atherosclerosis of lower extremity arteries as per laser Doppler flowmetry. Regionarnoye krovoobrashcheniye $i$ mikrotsirkulyatsiya. 2017; 16(4-64): 35-41 (in Russ.)]

5. Гавриленко А.В., Кравченко А.А., Шаталова Д.В. Повторные реконструкции после эндоваскулярного вмешательства у больной с критической ишемией нижних конечностей. Ангиология и сосудистая хирургия. 2017; 23(4): 118-121. [Gavrilenko A.V., Kravchenko A.A., Shatalova D.V. Secondary reconstructions after endovascular treatment of a woman with critical lower limb ischaemia. Angiologiya i sosudistaya khirurgiya. 2017; 23(4): 118-121 (in Russ.)]

6. Гавриленко А.В., Омаржанов О.А., Куклин А.В. Прогностическая ценность функционального резерва микроциркуляции и воспалительно-некротической реакции для определения тактики хирургического лечения больных с критической ишемией нижних конечностей. Анналь хирургии. 2005; 5: 23-28 [Gavrilenko A.V., Omarzhanov O.A., Kuklin A.V. Predictive value of the microcirculatory functional reserve and an inflammatory-and-necrotic reaction for determination of surgical treatment policy in patients with critical ischemia of the lower extremity. Annaly khirurgii. 2005; 5: 23-28 (in Russ.)]

7. Казаков Ю.И., Лукин И.Б. Система оценки состоятельности русла оттока для определения возможности выполнения бедренно-подколенного шунтирования в изолированный артериальный сегмент у больных с критической ишемией. Кардиология $u$ сердечно-сосудистая хирургия. 2014; 7(5): 30-34. [Каzakov Iu.I., Lukin I.B. Assessment of outflow channel for determination the possibility of the femoropopliteal bypass surgery in isolated arterial segment in patients with critical limb ischemia. Kardiologiya $i$ serdechnososudistaya khirurgiya. 2014; 7(5): 30-34 (in Russ.)]

8. Карпенко А.А., Стародубцев В.Б., Игнатенко П.В., Рабцун А.А., Митрофанов В.О. Результаты эндоваскулярных вмешательств у больных с окклюзионностенотическими поражениями артерий аортоподвздошного сегмента. Ангиология и сосудистая хирургия. 2016; 22(2): 77-84 [Karpenko A.A., Starodubtsev V.B., Ignatenko P.V., Rabtsun A.A., Mitrofanov V.O. Results of endovascular interventions in patients with occlusive stenotic lesions of arteries of the aortoiliac segment. Angiologiya i sosudistaya khirurgiya. 2016.22(2): 77-84 (in Russ.)]

9. Кордецки К., Мишаляк П., Левчук А., Новицки М., Завадски М., Мол М., Лукашевич А., Валецки Ж. Эффективность эндоваскулярного лечения окклюзионно-стенотических поражений подвздошных артерий при помощи самораскрывающегося стента Jaguar SM. Диагностическая и интервенционная радиология. 2011; 5(2): 35-43 [Kordetski K., Mishalyak P., Levchuk A., Novitski M., Zavadski M., Mol M., Lukashevich A., Valetski Zh. The effectiveness of endovascular treatment of occlusive-stenotic lesions of the iliac arteries with the help of self-opening stent Jaguar SM. Diagnosticheskaya $i$ interventsionnaya radiologiya. 2011; 5(2): 35-43 (in Russ.)]

10. Крупаткин А.И., Сидоров В.В. Функциональная диагностика состояния микроциркуляторно тканевых систем: колебания, информация, нелинейность: руководство для врачей. Москва: ЛИБPOKOM, 2013. 496 c. [Krupatkin A.I., Sidorov V.V. Functional diagnostics of microcirculatory tissue systems: oscillations, information, nonlinearity: a guide for physicians. Moscow: LIBROKOM, 2019. 496 p. (in Russ.)]
11. Лазаренко В.А., Бобровская Е.А. Оценка результатов бедренно-подколенного шунтирования у больных облитерирующим атеросклерозом с позиции микроциркуляции. Вестник Волгоградского государственного медицинского университета. 2016; 2(58): 67-69 [Lazarenko V.A., Bobrovskaya E.A. Evaluation of the results of femoral-popliteal bypass in patients with atherosclerosis obliterans from a microcirculation view. Vestnik Volgogradskogo gosudarstvennogo meditsinskogo universiteta. 2016; 2(58): 67-69 (in Russ.)]

12. Лазаренко В.А., Бобровская Е.А., Путинцева Е.В. Микроциркуляторные изменения у больных облитерирующим атеросклерозом при реконструкции аорто-бедренного артериального сегмента. Вестник экспериментальной и клинической хирургии. 2014; 7(4): 315-320. [Lazarenko V.A., Bobrovskaya E.A., Putintseva E.V. Microcirculatory changes in patients with obliterating atherosclerosis in the reconstruction of the aorto-femoral arterial segment. Vestnik eksperimental'noy i klinicheskoy khirurgii. 2014; 7(4): 315-320 (in Russ.)]

13. Национальные рекомендации по ведению пациентов с заболеваниями артерий нижних конечностей. Ангиология и сосудистая хирургия. 2013; 19(2): 1-75. [National guidelines for the management of patients with lower limb artery disease. Angiologiya $i$ sosudistaya khirurgiya. 2013; 19(2): 1-75 (in Russ.)]

14. Покровский А.В., Яхонтов Д.И. Значение оценки путей оттока при бедренно-тибиальных реконструкциях. Вестник Национального Медикохирургического Центра им. Н.И. Пирогова. 2013; 4: 18-24. [Pokrovskij A.V., Jahontov D.I. Role of venous cuff in femoral-tibial bypass surgery. Vestnik Natsional'nogo Mediko-khirurgicheskogo TSentra im. N.I. Pirogova. 2013; 4: 18-24 (in Russ.)]

15. Савельев В.С., Кузнецов М.Р., Вирганский А.О., Москаленко Е.П., Евграфов А.И. Физиологические аспекты диагностики пропускной способности микроциркуляторного русла нижних конечностей. Грудная и сердечно-сосудистая хирургия. 2002; 1: 31-37 [Savel'yev B.C., Kuznetsov M.R., Virganskiy A.O., Moskalenko E.P., Evgrafov A.I. Physiological aspects of diagnostics of capacity of microcirculatory bed of lower extremities. Grudnaya i serdechno-sosudistaya khirurgiya. 2002; 1: 31-37 (in Russ.)]

16. Стрельцова Н.Н., Васильев А.П., Бессонов И.С., Колунин Г.В. Изменение микроциркуляторной картины у больных перемежающейся хромотой после эндоваскулярного восстановления магистрального кровотока конечности. Регионарное кровообращение и микроциркуляция. 2018; 17(2): 42-48 [Streltsova N.N., Vasilyev A.P., Bessonov I.S., Kolunin G.V. The microcirculatory changes in patients with intermittent claudication after endovascular restoration of main blood flow to the extremity. Regionarnoye krovoobrashcheniye $i$ mikrotsirkulyatsiya. 2018; 17(2): $42-48$ (in Russ.)]

17. Федорович А.А., Багдасарян А.Г., Учкин И.Г., Соболева Г.Н., Бойцов С.А. Современные возможности неинвазивного контроля микроциркуляции и обмена веществ у человека. Ангиология и сосудистая хирургия. 2018; 24(1): 7-18 [Fedorovich A.A., Bagdasaryan A.G., Uchkin I.G., Soboleva G.N., Boytsov S.A. Present-day possibilities of non-invasive control over microcirculation and metabolism in man. Angiologiya $i$ sosudistaya khirurgiya. 2018; 24(1): 7-18 (in Russ.)]

18. Хабазов Р.И., Амиров Н.Ш., Амирова А.В., Јысенко Е.Р., Азарян А.С., Троицкий А.В. Отдаленные ре- 
зультаты эндопротезирования и стентирования подвздошных артерий после гибридных вмешательств. Вестник Национального Медикохирургического Центра им. Н.И. Пирогова. 2016; 11(1): 22-26. [Habazov R.I., Amirov N.Sh., Amirova A.V., Lysenko E.R., Azarjan A.S., Troickij A.V. Long-term results after endoprosthesis replacement and stenting operations of the iliac arteries after hybrid interventions. Vestnik Natsional'nogo Mediko-khirurgicheskogo Tsentra im. N.I. Pirogova. 2016; 11(1): 22-26 (in Russ.)]
19. Rutherford R.B., Baker J.D., Ernst C., Johnston K.W., Porter J.M., Ahn S., Jones D.N. Recommended standards for reports dealing with lower extremity ischemia: revised version. I Vasc Surg. 1997; 26(3): 517-538.

20. Zierler R.E., Jordan W.D., Lal B.K., Mussa F., Leers S., Fulton J., Pevec W., Hill A., Murad M.H. The Society for Vascular Surgery practice guidelines on follow-up after vascular surgery arterial procedures. 7 Vasc Surg. 2018; 68(1): 256-284. DOI: 10.1016/j.jvs.2018.04.018.

Для цитирования: Лазаренко В.А., Бобровская Е.А., Бондарев Г.А. Состояние макрогемодинамики и микроциркуляции у пациентов с окклюзионно-стенотическим поражением магистральных артерий нижних конечностей при различных типах реваскуляризующих вмешательств. Курский научно-практический вестник «Человек и его здоровье». 2019;(1):14-22. DOI: 10.21626/vestnik/2019-1/02.

\title{
MACROHEMODYNAMIC AND MICROCIRCULATION STATE IN PATIENTS WITH OCCLUSIVE- STENOTIC LESIONS OF THE MAIN ARTERIES OF THE LOWER EXTREMITIES IN VARIOUS TYPES OF REVASCULARIZING INTERVENTIONS
}

\author{
( Lazarenko V.A., Bobrovskaya E.A., Bondarev G.A. \\ Kursk State Medical University (KSMU) \\ 3, K. Marx st., Kursk, Kursk region, 305041, Russian Federation
}

\begin{abstract}
Objective. The aim was to study the state of macrohemodynamics, microcirculatory bed and their relationship in patients with occlusive-stenotic lesions of the aorto-iliac-femoral arterial segment before and after revascularizing operations. Materials and methods. The results of the analysis of macrogemodynamic and microcirculatory bed state in 330 patients with obliterating atherosclerosis of lower limb arteries in various types of revascularizing interventions are presented: femoral-popliteal (group I, $\mathrm{n}=140$ ), aorto-femoral (group II, $\mathrm{n=97}$ ) bypass surgery, angioplasty with iliac artery stenting (group III, $\mathrm{n}=93$ ). Results. There was a decrease in microcirculation in group I - 2.39 times, $p<0.001$, II -3 times, $p<0.001$, III -2.31 times, $p<0.001$, capillary blood flow reserve - in group I 1.63 times, $\mathrm{p}<0.001$, II -2.18 times, $\mathrm{p}<0.001$, III -1.77 times, $\mathrm{p}<0.001$ and an increase in arterial-venular bypass regardless of the level of damage, as well as an oppression of endothelial mechanism and an increase in neurogenic mechanisms of regulation of microcirculation in all groups. Surgeries do not normalize microhemodynamics. Correlation interrelations of macrohemodynamics, changes in clinical status and microcirculation indices both in basal blood flow and under conditions of postocclusive hyperemia were established. Changes in clinical status after surgery and bypass surgery had a negative-oriented correlation in all study groups $(\mathrm{r}=-0.2-0.3, \mathrm{p}<0.05-0.01)$, and ankle-brachial index before and after surgery and RCC - the positive-oriented relationship $(\mathrm{r}=0.2-0.4, \mathrm{p}<0.05-0.001)$. Conclusion. The state of microcirculation affects the change in clinical status after surgery, in this regard, it is necessary to take into account the parameters of microcirculation disorders and the mechanisms of regulation of tissue blood flow in surgical revascularization.
\end{abstract}

Keywords: obliterating atherosclerosis of lower extremity arteries; revascularizing interventions; macrohemodynamics; microcirculatory bed; mechanisms of regulation of tissue blood flow; occlusive functional tests; laser Doppler flowmetry.

Lazarenko Viktor A. - DM, Professor, Rector, Head of Surgical Diseases Department of FPE, KSMU, Kursk, Russian Federation. ORCID iD: 0000-0002-2069-7701. E-mail: azaroks@mail.ru

Bobrovskaya Elena A. - PhD, Associate Professor, Associate Professor of Surgical Diseases Department of FPE, KSMU, Kursk, Russian Federation. ORCID iD: 0000-0003-3898-5522. E-mail: ea-bobrovskaya@yandex.ru (correspondence author)

Bondarev Gennady A. - DM, Associate Professor, Professor of Surgical Diseases Department of FPE, KSMU, Kursk, Russian Federation. ORCID iD: 0000-0002-6761-562X. E-mail: gennadiy bondarev@mail.ru

\section{CONFLICT OF INTEREST}

The authors declare the absence of obvious and potential conflicts of interest related to the publication of this article.

\section{SOURCE OF FINANCING}

The authors state that there is no funding for the study.

\section{CONFORMITY WITH THE PRINCIPLES OF ETHICS}

The study was approved by the Ethical Committee under Kursk state medical university (Protocol No. 1 of 21.01.2012). Each patient signed the individual consent to participate in the study.

For citation: Lazarenko V.A., Bobrovskaya E.A., Bondarev G.A. Macrohemodynamic and microcirculation state in patients with occlusivestenotic lesions of the main arteries of the lower extremities in various types of revascularizing interventions. Kurskiy nauchno-prakticheskiy vestnik "Chelovek i ego zdorov'ye" = Kursk Scientific and Practical Bulletin "Man and His Health". 2019;(1):14-22. DOI: 10.21626/vestnik/2019-1/02. 